Fecha de recepción: noviembre 2018 Fecha de aceptación: marzo 2019

Versión final: julio 2019

\section{Los medios digitales y la autogestión de saberes. Una experiencia pedagógica en la enseñanza del diseño}

Luciana Anarella *

Resumen: La propuesta de este texto apunta a indagar sobre las cualidades significativas de los espacios involucrados en la enseñanza universitaria en general y en la enseñanza del diseño en particular. Se busca poner de relieve el valor e incidencia de los medios digitales en el aprendizaje del sujeto, visualizándolos como nichos vacantes para ser ocupados en la planificación de procesos de enseñanza-aprendizaje.

Los medios digitales con sus diversos dispositivos construyen modos de apropiación del saber significativamente distintos de los modos de apropiación del saber desde los medios tradicionales para la educación formal.

Se parte de la hipótesis de que los medios digitales (con la amplia oferta de circuitos para la comunicación e información) son generadores de una modalidad de aprendizaje autogestionado que promueve recorridos personales hacia la construcción de un saber propio e irrepetible. Esta cualidad se contrapone al recorrido lineal y dirigido de los espacios académicos, que si bien incentiva el desarrollo personal está regido por pautas institucionales que acreditan el saber cómo saber científico.

De esta manera se pondrán en comparación las cualidades de los medios digitales como medios virtuales que esgrimen el espacio de lo cotidiano con las cualidades de los medios físicos y reales que imperan en el espacio académico. El límite de cada uno de estos espacios será el punto de partida para pensar en una posible interacción entre la inmersión de la academia con la emersión de lo cotidiano.

Palabras clave: Enseñanza - Diseño - Innovación - Espacios académicos - Virtualidad Medios digitales.

[Resúmenes en inglés y portugués en las páginas 44-45]

${ }^{(*)}$ Diseñadora Gráfica. Docente FADU UBA. Investigadora.

\title{
Introducción
}

Este texto parte de una serie de premisas en torno a, las condiciones contextuales en que transcurre la enseñanza del diseño, las características disciplinares que aportan un valor propio asociado a los modos de enseñanza y al estrecho vínculo entre el diseño y los medios digitales. 
Las condiciones contextuales están vinculadas a los modos de aprendizaje cotidiano: a los múltiples accesos a la información (Google, Instagram, Pinterest, YouTube, entre otros), a los efímeros diálogos de comunicación (protagonizados por las redes sociales entre ellas Facebook y Twitter) y a la autonomía y practicidad del aprendizaje por tutoriales. Por otra parte las características disciplinares (del Diseño en general y el Diseño Gráfico en particular) aportan un valor propio asociado a la construcción del saber colectivo y colaborativo que constituye un modo particular de inteligencia para el desarrollo, producción y puesta en valor del proyecto de diseño.

A su vez el desarrollo tecnológico para los medios de comunicación opera como pilar de la educación, se establece una interacción dinámica mediada por dispositivos que amplía las posibilidades de acceso a la información y por lo tanto al desarrollo personal. Hoy, esos dispositivos digitales intervienen tanto como herramientas y materia prima para la construcción y puesta en valor de un proyecto de diseño; como herramientas y materia prima para construcción y puesta en valor de la comunicación cotidiana ya sea superflua o profunda.

Este texto es una reflexión sobre la posibilidad de conjugar los modos de aprendizaje en el espacio académico con los modos de aprendizaje en el espacio de lo cotidiano, intentado visualizar las cualidades de cada uno de ellos y dejando al margen los presupuestos que banalizan el aprendizaje auto gestionado y ponderar, por su parte, el saber propio e independiente.

Para ello se buscó indagar a partir de una experiencia pedagógica que pone en relieve alguna de las virtudes que emergen de las redes de comunicación como medio de aprendizaje autogestionado (en este caso Facebook) y su consonancia con el medio de aprendizaje institucionalizado (en este caso, el taller de Diseño de la carrera de Diseño Gráfico, FADU, UBA).

\section{La enseñanza académica y el cotidiano digital}

Tradicionalmente la educación formal es un espacio que tiende a descuidar el vínculo con el espacio de la educación no formal; se constituye como una burbuja poco permeable con lo cual el espacio académico se aísla del espacio en que los estudiantes interactúan cotidianamente.

Son momentos donde la comunicación se expande, los medios se multiplican e interactúan; vivimos inmersos en una cultura digital en pleno desarrollo. Muchos discursos se gestan desde estos códigos y es en el acceso y conocimiento de ellos, donde comienza el proceso de desarrollo de la educación. A su vez el contexto social que emerge desde las redes sociales, los dispositivos digitales para la comunicación y hasta el aprendizaje por tutoriales (que a su vez se alinean con el concepto de saber colectivo y colaborativo) constituye, hoy, la trama del aprendizaje cotidiano que construye una "matriz de aprendizaje" es decir "(...) un modelo o matriz de contacto con el mundo con la cual construimos hábitos de aprendizaje y maneras de percibir secuencias de conductas" (Quiroga, 1992). Nuestro cotidiano está impregnado de narraciones como "formas para darle forma al mundo" (Cyrulnik, 2002), narraciones como puntos de convergencia de la comunicación. Una comunicación que se propaga en la multiplicación de medios de diferentes caracterís- 
ticas tecnológicas (web, redes sociales, realidad virtual, realidad aumentada; que se suman a libros, diarios y revistas impresos, entre otros) y que convergen en un denominador común: la construcción de los nuevos modos de comunicación y representación visual.

En este contexto de convergencia, los dispositivos de diferentes tecnologías se reformulan y se retroalimentan pidiendo una revisión constante de su modo de lectura y usabilidad. Claro que los objetos no desparecen; mutan, se revalorizan y modifican su valor simbólico. Como bien expresa David Buckingham:

... la historia de la innovación indica que los nuevos medios no reemplazan necesariamente a los anteriores, sino que se suman al rango de opciones disponibles. En el proceso pueden modificar los motivos por los que las personas utilizan los medios de los que disponen, las clases de personas que los usan o los contextos en los que lo hacen. Pero al menos en la esfera de la cultura y las comunicaciones, las tecnologías normalmente se complementan entre sí de modos complejos y a veces imprevistos (Buckingham, 2008).

El grado de usabilidad de los diferentes dispositivos se mide en la observación empírica de los circuitos de comunicación que operan en los espacios educativos en general y en la enseñanza del Diseño en particular. De allí se prefigura el nivel de protagonismo de estos dispositivos (dinámicos y cambiantes) en el quehacer cotidiano de los estudiantes. La incorporación de estas tecnologías digitales provoca cambios en los hábitos y prácticas cotidianas promoviendo nuevos modos y vínculos comunicacionales tanto en las acciones, que estos dispositivos permiten, como en la construcción del discurso implícito en dichas acciones. Es decir que el soporte digital modifica hábitos tanto gestuales como de procedimiento, considerando que desde el momento que programamos nuestro día estamos manejando soportes digitales que codifican nuestras acciones en formas impersonales o universales quitándoles rasgos particulares (una muestra significativa es la universalización del emoticón).

Por su parte Pierre Levy junto con Michel Authier, acuñan el concepto de inteligencia colectiva que condensan con el término "cosmopedia". $\mathrm{La}$ "cosmopedia" refiere a "un nuevo tipo de organización de los conocimientos que reposa ampliamente en las posibilidades abiertas por la informática para la representación y la gestión dinámica de los conocimientos" (Lèvy, 2004).

La evaluación de Lèvy sobre la gestión de conocimientos en el espacio de la "cosmopedia" propone pensar que:

Más que a un texto de una sola dimensión, o incluso a una red hipertextual, estamos frente a un espacio multidimensional de representaciones dinámicas e interactivas.

Al cara a cara de la imagen fija y del texto, característico de la enciclopedia, la cosmopedia opone a un gran número de formas de expresión: imagen fija, imagen animada, sonido, simulaciones interactivas, mapas interactivos, sistemas expertos, ideografías dinámicas, realidades virtuales, vidas artificiales, etcétera. En última instancia, la cosmopedia contiene tantas semióticas y tipos 
de representaciones como se pueden encontrar en el mundo mismo. La cosmopedia multiplica los enunciados no discursivos.

A nuestro entender, la característica principal de la cosmopedia, y lo que le da su valor, es precisamente la no separación. Para los intelectos colectivos el conocimiento es un continuum, un gran mantel de retazos del cual cada punta puede plegarse sobre cualquiera otra. La cosmopedia desmaterializa las separaciones entre los conocimientos. Disuelve las diferencias entre las especialidades en tanto que son territorios donde se ejercen poderes, para solo dejar subsistir zonas de contornos difusos, estructuradas por conceptos de alcance variable y objetos en redefinición permanente. A una organización rígida de los conocimientos de disciplinas discretas y jerarquizadas (típico del territorio) $-\mathrm{o}$ al desmembramiento caótico de las informaciones y datos (típico de la mercancía) - se le substituye entonces una topología continua y dinámica.

Los miembros de una comunidad pensante indagan, inscriben, conectan, consultan, exploran. Su conocimiento colectivo se materializa en una inmensa imagen electrónica pluridimensional, en perpetua metamorfosis, agitándose al ritmo de las invenciones, de los descubrimientos, casi viva.

La cosmopedia pone no solo a disposición del intelecto colectivo el conjunto de conocimientos disponibles y pertinentes para él en un momento dado, sino que se ofrece igualmente como un lugar mayor de discusión, de negociación y de elaboración colectiva. Al ser imagen plural del conocimiento, la cosmopedia es el tejido mediador entre el intelecto colectivo y su mundo, el intelecto colectivo y él mismo. Los conocimientos ya no están separados de las cuestiones concretas que le dan sentido, ni de las prácticas que los engendran y que ellos modifican a su vez. Según las zonas de utilización y los trayectos de exploración, las jerarquías se invierten entre utilizadores y conceptualizadores, autores y lectores. En la cosmopedia, toda lectura es una escritura. La cosmopedia es como un espacio relativista sometido por la consulta y la inscripción. La inscripción propiamente dicha hace "cirugía" (cortes, costuras, trasplantes, operaciones discontinuas en general); la consulta, sin embargo, equivale a un masaje/doblado del espacio (inflexiones, operaciones continuas). Las preguntas, las interrogaciones sin respuesta ponen en tensión al espacio cosmopédico, señalan las zonas que llaman a la invención, a la innovación.

La cosmopedia, espacio continuo de las proximidades, implica primeramente en su estructura dinámica los vínculos, lazos y relaciones entre los enunciados. La situación, el contexto, los confines de una proposición no tienen que ser explicitados por discurso ya que están implicados en la forma moviente de la imagen. El contexto, las referencias, las coherencias conceptuales o prácticas de cada proposición no hay que recordarlas más puesto que están ya ahí, y la manera en que una nueva proposición se vincula al contexto (a otras proposiciones o a partes enteras de la cosmopedia) es materializada por posiciones relativas, proximidades, colores, luminosidades.

El intelecto colectivo forma, modela, pule, esculpe la imagen de su conocimiento y de su mundo más que traducirlo discursivamente. Repitámoslo, la 
simplificación resulta de la reducción considerable de la parte de texto en la exposición del conocimiento gracias a la implicación de las informaciones sobre las relaciones en la forma misma del "espacio-cosmopedia" (Lèvy, 2004).

Este intenso vínculo de la inmersión digital con el espacio cotidiano, invita a pensar que una enseñanza de calidad en el espacio académico debería apuntar a una mirada sobre el sujeto, su independencia y autonomía y no a la educación con una única mirada sobre el conocimiento de los objetos; es decir "a la formación de sujetos, de personas capaces de construir de manera libre y autónoma su propia subjetividad y de participar como actores protagónicos..." de la educación (Tedesco, 2014). Es decir, apelando a una construcción libre y autónoma que contemple el concepto sobre la libertad personal, entendida como:

La capacidad para definir orientaciones, valores, concepciones del mundo y de la vida que se apoyan en el dominio de los código por los que circula la información, en el conocimiento de la historia, en el manejo del razonamiento lógico, en el dominio de las formas de comunicación y de expresión artística, que solo pueden adquirirse sólidamente con procesos educativos sistemáticos (Tedesco, 2014).

Esta mirada sobre la autonomía del sujeto de aprendizaje se entiende como un mecanismo de reafirmación que otorga lugar al trabajo colectivo y universal y no a un mecanismo que sumerge al sujeto en el individualismo. Para lo cual es posible considerar tres aspectos que Tedesco destaca para la formulación de estrategias pedagógicas que den cuenta de una mirada universal de la educación y que implican trabajar sobre el desarrollo como sujeto (tanto desde el punto de vista curricular como desde las modalidades de enseñanzaaprendizaje, de la formación docente y de la integración de equipos):

a. la capacidad de formular un proyecto,

b. la capacidad de elaborar una narrativa acerca de la situación que se vive y acerca de las estrategias de acción que desarrolla cada actor, y

c. la confianza para enfrentar los desafíos que presentan las situaciones de aprendizaje.

En consonancia con los lineamientos de Tedesco, Boaventura de Sousa Santos en Conocer desde el sur: para una cultura política emancipatoria , señala algunos "campos sociales", que propician y a su vez enmarcan la construcción del saber, caracterizados por las acciones, las experiencias y las prácticas independientes. Entre ellos se destacan:

- Las experiencias de comunicación e información acerca de diálogos y conflictos posibles, derivados de la revolución de las tecnologías de comunicación y de información, entre los flujos globales de información y los medios de comunicación social globales, por un lado y, por otro, las redes de comunicación independientes transnacionales y los medios independientes alternativos.

- Las prácticas sociales y sus agentes que, en tanto todas las prácticas sociales se basan en conocimientos son, también, prácticas de saber. Sin embargo, al incidir sobre las 
prácticas, el trabajo de traducción intenta crear inteligibilidad recíproca entre formas de organización y entre objetivos de acción. En otras palabras, en este caso, el trabajo de traducción incide sobre los saberes en tanto que saberes aplicados, transformados en prácticas y materialidades.

Ambos señalamientos apuntan a enfatizar la necesidad de construcción de un discurso propio y comprender que "conocer es reconocer al otro como sujeto de conocimiento, es progresar en el sentido de elevar al otro del estatus de objeto al estatus de sujeto" (Santos, 2008), incorporando, interrelacionando y revalorizando el saber ajeno.

\section{La pedagogía de taller}

En la enseñanza del diseño, a diferencia de las materias humanísticas, la relación de los estudiantes con los contenidos disciplinares exige mucha producción visual, la cual por sus propias características, condiciona la modalidad y dinámica de las clases. Una diferencia significativa radica en la conformación del espacio físico: talleres de grandes dimensiones equipados con mesas (tableros) que se comparten entre 8 estudiantes aproximadamente y que se disponen en el aula de manera aleatoria sin un orden establecido; y que acentúa fuertemente la diferencia con las habituales aulas de clases teóricas donde el equipamiento, compuesto por sillas ordenas y orientadas a un frente donde se ubica el profesor, asigna un adelante, atrás, derecha e izquierda con respecto a la ubicación de los estudiantes en el espacio de estudio. Esta relación espacial implica un cambio radical en la mirada sobre las relaciones entre los estudiantes y profesores y por ende propicia un vínculo distinto entre los saberes, el estudiante y el profesor.

En este contexto "de taller" las dinámicas de clase transcurren, habitualmente, con la exposición de los trabajos producidos por los estudiantes y el debate sobre los mismos destacando aciertos y desaciertos en cada uno de los proyectos. Esta modalidad, propia de las carreras proyectuales, tiende a tener algunas variaciones en relación a la manera de debatir sobre los trabajos producidos, generando dinámicas donde habitualmente se pueden visualizar:

- Situaciones donde el profesor escucha a cada estudiante de manera individual y opina sobre el proyecto producido (relación unilateral e individual, al estilo consultoría).

- Situaciones donde el profesor marca errores y aciertos en cada uno de los trabajos frente a todo el grupo de estudiantes (relación unilateral y grupal).

- Situaciones donde el profesor frente a todo el grupo propone trabajar en problemáticas comunes tomando como ejemplo algún proyecto; poniendo en juego la posibilidad de trasferir problemáticas de un proyecto a otro (relación unilateral grupal con intervenciones o no según las posibilidades de diálogo que abra).

- Situaciones donde los estudiantes trabajan con sus proyectos analizando los problemas que puedan surgir y luego exponen lo analizado (relación grupal guiada por el docente).

- Situaciones donde el profesor construye preguntas para generar el debate y luego concluir en la verificación de contenidos trabajados (relación dialogal grupal coordinada por el docente). 
Cada una de estas dinámicas dan mayor o menor protagonismo al profesor, al estudiante y por consiguiente al saber. En mayor medida las 3 primeras modalidades son las que imperan destacando aciertos y desaciertos (en las producciones de los estudiantes), lo cual implica caer en el denominador común de entender al producto como el objeto de estudio, perdiendo de vista que "el objeto no es solo objeto sino, al mismo tiempo, problema: lo que está enfrente, como obstáculo e interrogación" (Freire, 2005).

Las formas de "colonialismo" (en términos de Freire) que se evidencian en las carreras proyectuales no se visualizan como una re producción de teorías y saberes construidos desde la palabra sino como una re producción de material visual según tendencias, modas y estéticas guiadas por los parámetros que fluyen de la comunicación "globalizada". Se puede decir que se establece entre estudiantes, profesores y saber una relación "bancaria" que "pretende mantener la inmersión" del sujeto, dejando de lado la posibilidad de "buscar la emersión de las conciencias, de la que resulta la inserción critica” (Freire, 2005). Y que, por otra parte, acentúa el sentido lineal de la educación como una

Totalidad hecha de partes homogéneas, que deja por fuera, ignora o trata de subsumir a su lógica y reglas de constitución gran parte de la realidad, (...) desperdiciando experiencias sociales (...) desdibujando sus potencialidades críticas y transformadoras, convirtiendo el futuro en destino inalterable y ya dado (Suarez, 2008).

Pero en un contexto de convergencia de tecnologías y redes virtuales, y desde una mirada crítica

La realidad, como quiera que se la conciba, es considerada como un campo de posibilidades (...) como el análisis crítico de lo que existe que reposa sobre el presupuesto de que los hechos de la realidad no agotan las posibilidades de la existencia, y que, por lo tanto, también hay alternativas capaces de superar aquello que resulta criticable en lo que existe. El malestar, la indignación y el inconformismo frente a lo que existe sirven de fuente de inspiración para teorizar sobre el modo de superar tal estado de cosas (Santos, 2008).

En consiguiente trabajar en nuevas propuestas pedagógicas, no implica solo conceptualizar sobre los contenidos disciplinares de la práctica académica sino también intervenir sobre aquellos contenidos que están implícitos en la práctica cotidiana. Visualizar dichas prácticas permite reiniciar sus significados como saberes que surgen de lo cotidiano y ponderar la idea de que

Ya nadie educa a nadie, así como tampoco nadie se educa a sí mismo, los hombres se educan en comunión, mediatizados por el mundo (...) No se trata sólo de saber cómo es el alumno, sino de valorar su conocimiento, su cultura. Tanto cuando está adherida al pensamiento mágico, como cuando contiene núcleos de buen sentido, como cuando posee un plus de conocimiento (Hillert, 2008). 
Esta concepción sobre la enseñanza se nutre de una mirada propia sobre la disciplina, porque entiendo que la acción planificada opera como tutor de la práctica del diseño pero se retroalimenta de las acciones y saberes cotidianos que surgen tanto de los entornos reales como de los entornos virtuales.

La planificación es un espacio consecutivo al espacio de lo espontáneo y como tal implica una trama estratégica desde una mirada consciente -al menos, en algunos aspectos- de la textualidad en la que van a insertarse. Estos textos de lo cotidiano son dinámicos y fluyen entre las múltiples interacciones con que se construyen (Anarella, 2008).

Por lo tanto la enseñanza del diseño no puede estar ajena a la movilidad de lo cotidiano y por lo tanto de la mutación fluctuante de la disciplina.

Dice Pierre Lèvy:

Vivimos en miles de espacios diferentes, cada uno con su sistema de proximidad particular tal (temporal, afectivo, lingüístico, etcétera), que una entidad cualquiera puede estar cerca de nosotros en un espacio y muy alejada en otro. Cada espacio posee su axiología, su sistema de valor o de medida particular. El objeto tal, que será muy "pesado" en cierto espacio, será ligero, marginal, en otro. Una buena parte de nuestra actividad cognitiva consiste en distinguirnos entre la multitud de "mundos" diferentes en los cuales navegamos. Debemos descubrir rápidamente la topología y la axiología de los nuevos espacios en los cuales debemos participar, no confundir los sistemas de valores, apreciar la evolución de las situaciones (Lèvy, 2004).

Es decir los circuitos, los medios y las redes globales de información y comunicación son espacios de construcción, son nichos alternativos de construcción del saber permeables de ser detectados y visibilizados (como deconstrucción) para analizar y cuestionar; en términos de J. Derrida, "parejas de conceptos que se aceptan normalmente como evidentes y naturales, que parece como si no se hubieran institucionalizado en un momento preciso" y evitar que "a causa de esta naturalidad adquirida, semejantes oposiciones limiten el pensamiento” (J. Derrida, 1986).

\section{La pedagogía de lo cotidiano}

Partiendo del análisis de la práctica docente y de la práctica cotidiana, de inmersión en los medios digitales como el espacio de la "cosmopedia", surgen algunas preguntas recurrentes: ¿Cuál es la contribución de los medios digitales en la enseñanza del diseño? ¿Cómo capitalizar esas prácticas y permitir sus aportes, legitimándolas como irrupciones innovadoras que apuntan a la independencia del sujeto? ¿Cómo construir hoy una pedagogía que conjugue la enseñanza en el espacio académico con la enseñanza en el espacio cotidiano? 
¿Es el entramado de redes sociales digitales el medio para la fusión entre la academia y el cotidiano? ¿Cuáles son los aportes de cada uno de los entornos (real y virtual)?

Una experiencia realizada en el taller de Diseño, de la Carrera de Diseño Gráfico, FADU, UBA; apunta, justamente, a poner de relieve "la comprensión de las relaciones que existen en la sociedad entre lo particular y el todo, entre lo específico y lo universal" (Henry Giroux, 1992).

Lo particular y el todo, lo específico y lo universal se tensionan en la búsqueda de alternativas que pongan en juego las relaciones entre los distintos espacios de comunicación de lo académico y los espacios de comunicación de lo cotidiano. Las redes sociales, entre otros, son espacios donde lo específico convive con lo universal y lo particular convive con el todo.

Dicha experiencia (transitada en el taller de Diseño, como experiencia pedagógica) supuso generar un espacio de trabajo virtual auto gestionado por un grupo de aproximadamente 110 estudiantes. Se propuso usar un grupo de facebook para que cada estudiante suba su producción en base a una consigna dada y que los mismos estudiantes cuestionen, evalúen, propongan, recomienden y debatan sobre el trabajo de sus compañeros.

Se generó un espacio donde se ponen en juego los saberes particulares y los saberes académicos sin la mediación del profesor. Un espacio donde los estudiantes intercambian opiniones sobre la producción de sus trabajos sin la participación de los profesores quienes se comportan como veedores de los debates. Los debates generados en ese espacio resultan un material para la reformulación y evaluación crítica sobre las modalidades de enseñanza con que se trabaja en el taller y son un material para visualizar aquellos saberes de lo cotidiano que se ponen en juego sin la mediación del profesor, de allí su carácter pedagógico. Si bien la propuesta es muy simple y pareciera que se asocia a otras modalidades habituales, es en su análisis donde radica el mayor interés.

Transcurrida la experiencia, detectamos principalmente tres cualidades de esta modalidad de trabajo, tanto del ámbito relacional como del ámbito disciplinar:

- Visualizamos que aquellos estudiantes que tenían poca participación en el espacio presencial (con la coordinación del docente), en el espacio virtual tomaban otro protagonismo dando cuenta de una postura crítica que el espacio físico no le permitía desarrollar.

- Detectamos como algunos contenidos trabajados en el taller no eran lo suficientemente comprendidos a pesar del abordaje reiterado y el uso de las preguntas para profundizar sobre el tema.

- Detectamos contenidos que emergen desde las interpretaciones personales de los estudiantes y que no fueron tenidos en cuenta en la programación del ejercicio.

Esta experiencia permite, a su vez, visualizar varios aspectos que están alineados y que intentaré hilar para encontrar nexos entre la relación que se establece entre el espacio académico y el espacio cotidiano, entre el espacio físico y el espacio virtual, entre el espacio particular y el espacio general.

Se observó (en los debates e intervenciones que se generaron en el grupo) que se han multiplicado las posibilidades de aprendizaje, tanto por la misma práctica de intervención como por la aceptación de que es un espacio habilitado para el error. A su vez, la reflexión 
sobre las producciones, la autoevaluación y la autogestión del grupo son fruto de una comprensión natural sobre el funcionamiento del espacio virtual, el cual abre paso a las argumentaciones espontáneas y a la participación desde la ausencia corporal.

Estas cualidades prefiguran lo que entiendo como pedagogía de lo cotidiano, una pedagogía que involucra un espacio para el hacer/pensar/participar desde el anonimato de la ausencia corporal y que otorga una autonomía y libertad de construcción del saber al resguardo de la mirada sentenciosa del experto. Una pedagogía que marcan una diferencia enriquecedora y resulta un interesante insumo para la construcción de una mirada sobre la enseñanza que integre el espacio cotidiano con el espacio académico.

Deconstruir el espacio académico y el espacio cotidiano desde sus propiedades permite visualizar los componentes que inciden tanto en la enseñanza como en el aprendizaje. En dicho análisis se definen binomios que permiten caracterizar y a la vez confrontar dichas propiedades formativas. Se define que, en tanto el espacio académico se conjuga como un espacio institucional, cerrado, gestionado, evaluado y presencial; el espacio cotidiano se conjuga como un espacio propio, abierto, autogestionado, autoevaluado y virtual.

\section{- Espacio institucional/espacio propio}

El espacio académico está fuertemente influenciado por ser de carácter institucional. Promueve vínculos de aspecto ideológico y social mediados por la propia institución (estatal o privada). Es un espacio donde el aprendizaje se ve condicionado por las cualidades de habitabilidad, por su arquitectura, mobiliario e instrumentos a disposición, que permiten un tipo específico de prácticas.

El espacio cotidiano es un espacio de carácter propio que se sumerge en un terreno de relaciones sociales significativamente distintas. La impronta de lo cotidiano modifica la relación tiempo - espacio - sociedad con condiciones de habitabilidad que se desdibujan en la misma práctica. Es un espacio propio e intangible que promueve prácticas y aprendizajes independientes.

\section{- Espacio cerrado/espacio abierto}

El espacio académico es un espacio cerrado que contiene al saber legitimado, que genera un círculo acotado de relaciones y que establece grupos de pertenencia. Es un espacio inamovible, de saberes disciplinares poco cambiantes y que interactúan en sus propios límites argumentativos guidados por respuestas esperables que construyen un circuito cerrado de saberes.

El espacio cotidiano por el contrario, es un espacio abierto dinámico y cambiante donde se desdibujan los límites argumentativos del saber académico. Es un espacio donde el saber cotidiano está mediado por infinidad de interacciones multidisciplinarias regidas por la expectativa de lo inesperado. El saber cotidiano es un espacio sin límites que permite hacer y deshacer desde múltiples abordajes.

\section{- Espacio gestionado /espacio autogestión}

El espacio académico es un espacio gestionado, de administración de saberes que apuntan a un fin específico a corto o largo plazo; es un espacio de orden jerárquico, conducido por el docente/cátedra el cual genera pautas de producción y modos de apropiación que, en 
tanto planificados y controlados, pretenden ser respetados con condiciones mínimas de cumplimiento en relación a los contenidos, al tiempo y al nivel de producción.

El espacio cotidiano, por su parte, es un espacio de autogestión de la producción y del tiempo; pero a su vez, es un espacio de autogestión de los saberes y las prácticas; no tiene un fin necesariamente específico y deja abierta la posibilidad de reformulación de los mecanismos de producción. Busca la verificación o refutación de las hipótesis que la propia planificación pone en juego pero, a su vez, es un espacio que pone en alerta la rigidez de lo planificado apostando a la versatilidad de lo imprevisto.

\section{- Espacio de evaluación / espacio de autoevaluación}

El espacio académico es un espacio de evaluación guiado por parámetros estipulados, tanto para los contenidos y objetivos disciplinares como los contenidos y objetivos pedagógicos. Es un espacio de evaluación permanente, de competencia y de comparación, donde se establece una relación asimétrica de poder que genera estados de dependencia. En este espacio, el valor del saber está regido por los saberes acreditados por la propia institución, las autoridades y los docentes a cargo. Es decir, es un espacio para la acreditación de saberes. El espacio cotidiano, por su parte, es un espacio de autoevaluación que no persigue la acreditación sino la construcción de un saber propio, particular e irrepetible. El valor del saber académico queda relegado al saber de la práctica cotidiana, a la independencia del sujeto y a la valoración del saber individual generado desde el mismo espacio cotidiano. Se autoevalúan no solo las producciones sino los comportamientos de los diferentes actores desde una relación de paridad que estimula la independencia.

\section{- Espacio presencial/ espacio virtual}

El espacio académico es un espacio presencial, de vínculos sociales atravesados por un interés común (las disciplinas que los convoca) que opera como motor y materia prima. Es un espacio de intercambio cuerpo a cuerpo con condicionantes propios de la presencia, que inciden en cada individuo de manera aleatoria según sus características.

Es un espacio que, en tanto presencial, se desarrolla en un tiempo lineal y continuo, que pretende un estado de atención permanente.

El espacio cotidiano es un espacio virtual donde la acción se vuelve íntima, individual y a su vez pública; opera en el anonimato del cuerpo y permite deshacer los límites de lo presencial. El motor de los intereses no es necesariamente común y se multiplica en el espacio virtual porque la diversidad del medio lo habilita. El tiempo no es lineal ni continuo, por el contrario es variable y discontinuo.

En consecuencia y considerando las cualidades de cada uno de los espacios, podremos pensar e indagando sobre los modos de fusionar o complementar la pedagogía de la academia con la pedagogía de lo cotidiano y visualizar los tipos de aprendizaje que entre tanto se ponen en juego:

- Aprendizaje desde la práctica, en tanto la misma práctica implica la toma de decisiones para la formulación de proyectos.

- Aprendizaje desde el error, sorteando lo inesperado y resolviendo desde la autogestión 
- Aprendizaje desde la problematización frente a la relación entre lo presencial y lo virtual, resolviendo nexos poco visibles en la planificación.

- Aprendizaje desde las argumentaciones espontáneas, enfrentando las preguntas y respuestas sobre las producciones, algunas deseables otras impredecibles.

- Aprendizaje en la reflexión sobre los resultados, reflexiones que surgen en el instante o a posteriori, reflexiones atemporales.

- Aprendizaje desde la autoevaluación de la práctica; instancia donde se involucran y entrelazan criterios personales. Es la evaluación de los logros personales, los temores, la puesta en juego de los saberes propios que genera la confianza para enfrentar los desafíos que presentan las situaciones de aprendizaje.

\section{A modo de cierre}

Así planteado, la interacción entre espacios y la visualización de los diversos tipos de aprendizajes, deberían conducir a transformar y mejorar las formas de aprehender la realidad en general y del diseño en particular.

Hoy la tecnología nos permite trabajar en espacios virtuales que trascienden los límites de las aulas, habilitando modos de participación poco aprovechados como instrumentos para una nueva pedagogía en pos de un pensamiento que confronte una pedagogía de la respuesta con una pedagogía de la pregunta. Es decir que confronte la idea del saber cómo una respuesta certera y cerrada con la idea del saber cómo una pregunta para la problematización y apertura, "la primera (versión bancaria), pretende mantener la inmersión, la segunda por el contrario, busca la emersión de las conciencias, de la que resulta su inserción critica en la realidad" (Freire, 2005).

Esta experiencia en el taller de Diseño es una propuesta que surge en busca de pedagogías que permitan la construcción y fortalecimiento de saberes propios, sujetas a modificaciones y reformulaciones que, por su carácter crítico, contemple variables sobre el intercambio de relatos y prácticas que se retroalimentan en la dinámica misma de la vida social. Esto,

Supone avivar la imaginación pedagógica y la creatividad cultural y dirigirlas hacia el diseño y puesta en marcha de modalidades, estrategias y dispositivos de trabajo que planteen relaciones horizontales (...) que hagan ganar en inteligibilidad a todos los participantes (...), que reconozca y respete diferencias sin tratar de colonizarlas en nombre de una validez científica... (Suarez, 2008).

La frontera entre la academia y lo cotidiano, es un terreno aun poco transitado, cargado de prejuicios y condicionamientos que derivan de nuestra propia formación como formadores y como profesionales. Indagar, proponer y generar bisectrices que establezcan nexos entre ambos espacios, sería un aporte para profundizar en los modos de aprendizaje desde dispositivos que tanto aportan al aprendizaje cotidiano pero que aún están vedados en el aprendizaje académico. 
Finalmente y en palabras de Lidia Rodriguez rescato el sentido del diálogo para la construcción del saber propio, tanto en la enseñanza como en el aprendizaje, tanto en el espacio académico como en el espacio cotidiano.

A dialogar se aprende, al igual que se aprende a escuchar y a hablar. Se aprende a reconocer en el otro un igual. "Poder decir: no se" es un acto que tiende un puente con el otro, y por todo eso, profundamente formativo.

(Rodriguez, 2008)

\section{Bibliografía}

Aguerrondo, I. (2005). Estrategias para mejorar la calidad y la equidad de la educación en la Argentina, en ¿Cómo superar la desigualdad y la fragmentación del sistema educativo argentino? Buenos Aires: UNESCO.

Anarella, L. y otros (2008). Piquete de ojo, La semiosis de lo espontáneo y lo planificado en el contexto de la crisis. Buenos Aires: Ediciones FADU, Nobuko.

Buckingham, D. (2008). Más allá de la tecnología. Aprendizaje infantil en la era de la cultura digital. Buenos Aires: Ediciones Manantial.

Cyrulnik, B. (2002). El encantamiento del mundo. Barcelona: Gedisa.

Derrida, J. (1986). La metáfora arquitectónica. (Architetture ove il desiderio può abitare). Entrevista de Eva Meyer, Domus, 671, (pp. 16-24),.

Dubet, F. (2011). Repensar la justicia social. Buenos Aires: Editorial Siglo XXI.

Feldfeber, M. y Gluz, N. Las políticas educativas en argentina: herencias de los '90, contradicciones y tendencias de "nuevo signo" Educ. Soc., Campinas, v. 32, n. 115, p. 339-356, abr.-jun. 2011 Disponivel em http://www.cedes.unicamp.br.

Freire, P. (1975). Pedagogía del oprimido. Buenos Aires: Siglo XXI.

Gentili, P. Las deudas educativas del Bicentenario. Cuadernos del Pensamiento Crítico Latinoamericano $N^{o} 35$. CLACSO, octubre de 2010. Publicado en La Jornada de México, Página 12 de Argentina y Le Monde Diplomatique de Bolivia, Brasil, Colombia, Chile, España y Perú.

Giroux, H. (1992). Teoría y resistencia en educación. Una pedagogía para la oposición. México: Siglo veintiuno.

Gluz, N. y Rodríguez Moyano, I. Asignación Universal por Hijo, condiciones de vida y educación. Las política sociales y la inclusión escolar en la provincia de Buenos Aires. Archivos Analíticos de Políticas Educativas, 21 (21). Recuperado [data] http://epaa.asu. edu/ojs/article/view/1102, 2013.

Hillert, F. (2008). El giro copernicano pedagógico de Freire. Revista Novedades Educativas, Año 20, N²09. Novedades Educativas, Buenos Aires, .

Kessler, G. (2005). Exclusión social y desigualdad ¿nociones útiles para pensar la estructura social argentina? en ¿Cómo superar la desigualdad y la fragmentación del sistema educativo argentino? Buenos Aires: UNESCO.

Lèvy, P. Inteligencia colectiva. Por una antropología del ciberespacio. BVS (Biblioteca Virtual em Saüde) Washington, DC. Marzo de 2004. 
Quiroga, A. (1992). Matrices de aprendizaje. Constitución del sujeto en el proceso de conocimiento. Buenos Aires: Ediciones Cinco.

Rodriguez, L. (2008). Actualidad del pensamiento y la praxis freiriana. Revista Novedades Educativas, $\mathrm{N}^{\circ} 209$. Novedades Educativas, Buenos Aires.

Santos, B. de S. (2008). Conocer desde el Sur. Para una cultura y política emancipatoria. La Paz: Clacso.

Suarez, D. H. (2008). La tradición crítica en educación y reconstrucción de la pedagogía. Revista Novedades Educativas, Año 20, № 209. Novedades Educativas , Buenos Aires.

Suarez, D. H.; Ochoa, L. y Dávila, P. (2005). Documentación narrativa de experiencias pedagógicas. Revista Nodos y Nudos, Vol. 2; N 17. Universidad Pedagógica Nacional de Colombia (UPN), Bogotá.

Tedesco, J. C. (2005). Los nuevos temas de la "agenda" de la transformación educativa en Argentina en ¿Cómo superar la desigualdad y la fragmentación del sistema educativo argentino? Buenos Aires: UNESCO.

Tedesco, J. C. (2014). Educación y justicia social. Santiago: UNESCO.

\begin{abstract}
The proposal of this text aims to investigate the significant qualities of the spaces involved in university education in general and in the teaching of design in particular. It seeks to emphasize the value and impact of digital media in learning the subject, regarding them as vacant niches to be occupied in the planning of teaching-learning processes. Digital media with their various devices build modes of appropriation of the know significantly different from the modes of appropriation of knowledge from traditional media to formal education. The assumption is that digital media (with the wide range of circuits for communication and information) are generators of a mode of self-managed learning that promotes personal tours toward the construction of a know own and unrepeatable. This quality is opposed to the linear route and directed the academic spaces, that while encourages the development staff is governed by institutional guidelines that accredit the know how scientific knowledge. In this way will be compared the qualities of digital media as virtual media wield the space of the everyday with the qualities of the physical media and actual conditions in the academic space. The limit of each one of these spaces will be the starting point for thinking about a possible interaction between the immersion of the Academy with the rise of the everyday.
\end{abstract}

Keywords: Teaching - Design - Innovation - academic spaces - Virtuality - Digital Media.

Resumo: A proposta do presente texto tem como objetivo investigar as qualidades significativas dos espaços envolvidos no ensino universitário em geral e no ensino do design em particular. Procura-se enfatizar o valor e o impacto da mídia digital na aprendizagem do assunto, considerando-os como nichos vagos para ser ocupado no planejamento de processos de ensino-aprendizagem. Mídia digital com seus vários dispositivos construir modos de apropriação do saber significativamente diferente dos modos de apropriação de conhecimentos a partir da mídia tradicional à educação formal. A suposição é a de que a 
mídia digital (com a vasta gama de circuitos de comunicação e informação) são geradores de um modo de auto-gerida de aprendizagem que promove passeios pessoais para a construção de um saber próprio e irrepetível. Esta qualidade é contra a rota linear e dirigiu os espaços acadêmicos, que, enquanto encoraja o desenvolvimento pessoal é regido por diretrizes institucionais que Credenciar a saber como o conhecimento científico. Desta forma, serão comparadas as qualidades da mídia digital como a mídia virtual empunha o espaço do quotidiano com as qualidades do meio físico e das condições reais do espaço acadêmico. O limite de cada um desses espaços será o ponto de partida para pensar sobre uma possível interação entre a imersão da Academia com a ascensão do dia-a-dia.

Palavras chave: Ensino - Design - Inovação - espaços acadêmicos - A virtualidade - Mídia Digital.

[Las traducciones de los abstracts fueron supervisadas por el autor de cada artículo] 